\title{
Evidence Based Health Informatics: 10 Years of Efforts to Promote the Principle
}

\section{Joint Contribution of IMIA WG EVAL and EFMI WG EVAL}

\author{
M. Rigby', E. Ammenwerth ${ }^{2}$, M.-C. Beuscart-Zephir ${ }^{3}$, J. Brender ${ }^{4}$, H. Hyppönen ${ }^{5}$, S. Melia ${ }^{6}$, \\ P. Nykänen ${ }^{7}$, J. Talmon ${ }^{8}$, N. de Keizer ${ }^{9}$ \\ 1 Keele University, School of Public Policy and Professional Practice, Keele, United Kingdom \\ 2 UMIT, University for Health Sciences, Medical Informatics and Technology, Hall in Tyrol, Austria \\ ${ }^{3}$ INSERM-CIC-IT, CHU Lille, Lille, France \\ ${ }^{4}$ Aalborg University, Dept. of Health Science and Technology, and Vitual Center for Health Informatics, Aalborg, Denmark \\ ${ }^{5}$ National Institute for Health and Welfare, Information Department, Helsinki, Finland \\ ${ }^{6}$ Telefonica UK Ltd., Slough, United Kingdom \\ ${ }^{7}$ Univ. of Tampere, School of Information Sciences, Centre for Information and Systems, eHealth, Tampere, Finland \\ ${ }^{8}$ Maastricht University, School for Public Health and Primary Care: Caphri, Maastricht, The Netherlands \\ ${ }^{9}$ Academic Medical Center, Department of Medical Informatics, Amsterdam, The Netherlands
}

\begin{abstract}
Summary
Objectives: To present the importance of Evidence-based Health Informatics (EBHI) and the ethical imperative of this approach; to highlight the work of the IMIA Working Group on Technology Assessment and Quality Improvement and the EFMI Working Group on Assessment of Health Information Systems; and to introduce the further important evaluation and evidence aspects being addressed. Methods: Reviews of IMIA, EFMA and other initiatives, together with literature reviews on evaluation methods and on published systematic reviews.

Results: Presentation of the rationale for the health informatics domain to adopt a scientific approach by assessing impact, avoiding harm, and empirically demonstrating benefit and best use; reporting of the origins and rationale of the IMIA- and EQUATOR-endorsed Statement on Reporting of Evaluation Studies in Health Informatics (STARE-HI) and of the IMIA WG's Guideline for Good Evaluation Practice in Health Informatics (GEP-HI); presentation of other initiatives for objective evaluation; and outtining of further work in hand on usability and indicators; together with the case for development of relevant evaluation methods in newer applications such as telemedicine. The focus is on scientific evaluation as a reliable source of evidence, and on structured presentation of results to enable easy retrieval of evidence. Conclusions: EBHI is feasible, necessary for efficiency and safety, and ethically essential. Given the significant impact of health informatics on health systems, care delivery and personal health, it is vital that cultures change to insist on evidence-based policies and investment, and that emergent global moves for this are supported.
\end{abstract}

\section{Keywords}

Evidence-based health informatics; evaluation; evidence; health informatics; ethics

Yearb Med Inform 2013:34-46

\section{Introduction}

This paper outlines the role of Evidence-based Health Informatics, and sums up the current state of the art and techniques, particularly the crucial aspect of generating reliable scientific evidence through systematic evaluation. Evidence-based Health Informatics (EBHI) is the theme of this IMIA Yearbook for 2013. The term EBHI has belatedly emerged only in the last few years. However, the vision and ethical drive, and in turn the activities that commenced the development of the evidence base, and of the guidelines, methodologies, and repositories of evaluation and its resultant evidence, have a longer history dating back to the late 1980s. In later years, the establishment of the International Medical Informatics Association (IMIA) Working Group on Technology Assessment and Quality Improvement and the European Federation of Medical Informatics (EFMI) Working Group on Assessment of Health Information Systems (subsequently referred to as the IMIA and EFMI WGs) on this topic has created platforms that enable the further development of those activities.

Only recently has EBHI been firmly put on the international agenda in the form of the anticipated global collaboration between IMIA and the World Health Organisation (WHO) on this crucial principle [1]. Overall these steps mark an overdue recognition of the importance of emphasising a scientific, evidence-based, and ethically responsible approach to health informatics. They also place it firmly alongside all other health sciences in approach and responsibilities.

\subsection{The Goals of Evidence-based Health Informatics}

The core goals of Evidence-based Health Informatics (EBHI) are to ensure that policies and implementations are based on evidence (as opposed to the currently prevalent combination of aspiration and marketing promises); and to avoid iatrogenic or institutional damage caused by adverse selection or implementation of health informatics applications. Achievement of this requires the development and use of scientific approaches to obtaining the evidence. As indicated below a number of advocates have been pursuing this vision, which has then been facilitated by concerted action by the IMIA and EFMI Working Groups covering this responsibility.

It is right and proper that the health informatics community should lead this move to an evidence-based approach, and promote responsible and reasoned solutions. No longer is there a need to show that health informatics applications can be beneficial to health care delivery and to patients, but there is an overdue need to show how these can be optimised, to recognise that on occasions 
harm can be done, and thus to develop both the scientific culture and the methods to facilitate a scientific, evidence-based, and societally responsible position.

As has been stated previously, this links to an ethical responsibility to bring health informatics into the same scientific culture and rigour as other scientific applications in health [2 ]. One of the four core principles of health ethics is that of 'non nocere' not to harm [3], and no health informatics system can be seen to fulfil this principle unless it has been evaluated in practice, and in its current version. Furthermore, the Precautionary Principle would suggest that unless an application has been proven to be beneficial, or at very least not harmful, it should not be put into general use, and this principle for example underpins many European Commission policies $[4,5]$. By contrast, some health informatics systems have been proved in retrospect to be harmful $[6,7]$, but this has been proven only after damage has been done. Few applications are rigorously tested as meeting the Precautionary Principle, yet there is still an enduring reluctance to take an evidence-based approach to the degree that would be expected in any other new health intervention. Moreover, many of the evaluations performed have been summative studies, assessing the effect or outcome of the evaluation object at a certain point of time after implementation. In most cases, formative evaluation would be more beneficial, to evaluate throughout the system's developmental lifecycle and thus provide information for improving the system during development and early use.

Not only is an evidence-based approach needed in health informatics in order to comply with health ethical principles, but also to ensure an ethical commercial approach. Though business ethics is gaining recognition elsewhere, with its own dedicated journal and institute $[8,9]$, these are seldom talked of in the health informatics sector. But ethics permeates other areas of healthcare, and it is difficult to accept that health informatics should be exempted. Health informatics systems and individual applications by definition affect patients' lives and health professionals' livelihoods, and so the supplying vendors need to apply an evidence-based approach, and eschew the marketing of promises, vapour ware, and the assertion that things will work in the end $[2,10]$.

The latest revision of the EU Medical Device Directive (MDD) [11] that came into effect in early 2011 considers software for - among other purposes - the diagnosis, treatment, monitoring or alleviation of diseases and injuries as a medical device. Although there is still discussion on what Health Informatics applications are governed by this regulation, this change indicates that regulatory bodies have started to identify the need to require some proof of the safety of such applications before they can effectively be marketed in Europe. Meanwhile, in the United States, the Federal Drug Administration (FDA) is working with the U.S. Office of the National Coordinator for Health Information Technology to develop a Health IT Regulatory Framework, by means of the Food and Drug Administration Safety Innovation Act (FDASIA) of 2012.

Evidence-based Health Informatics should become pervasive in the thinking throughout the academic, application, policy and vendor communities within health informatics. The balance of this paper will give an overview of the principles, and the progress to date in its development in practice.

\subsection{History and Definition of Term EBHI}

The first published use of the term evidence-based health informatics was in the mid-1990s, in the setting of McMaster University, from where the initial Evidence-Based Medicine Working Group was supported, to describe information services that support evidence-based medicine [12]. The first cited absorption of this concept into the health informatics domain came at Medinfo in 2001, with recognition that "information systems are no different from any other health systems, in needing to be evidence-based, and specified and implemented based on best evidence-based information" [13].

Impetus to promote and implement evidence-based health informatics as a principle and a goal came in 2003, when it was developed and taken forward through an Exploratory Workshop on New Approaches to the Systematic Evaluation of Health Information Systems, organized by the University for Health Sciences, Medical Informatics and Technology (UMIT) in Innsbruck, Austria, and funded by the European Science Foundation. One output from this event was the Declaration of Innsbruck, which also represented the start of activities of the IMIA and EFMI WGs [14]. The lead Recommendation of this Declaration, further discussed below, was that "Evaluation should be seen as an ethical imperative", and it included the statement that evaluation studies are needed to "support reflective practice in health informatics in general, enabling the emergence of an evidence-based health informatics profession" [14]. In 2006, the IMIA Yearbook of Medical Informatics was devoted to the topic "Assessing Information Technologies for Health", and the term evidence-based health informatics was used in this context [15].

To the best of our knowledge, the first published definition of EBHI stems from 2010, suggesting that EBHI comprises the "conscientious, explicit and judicious use of current best evidence when making decisions about the introduction and operation of IT in a given health care setting" [16]; this definition follows closely from the definition of Evidence-Based Medicine (EBM) [17]. While EBM aims at integrating individual clinical expertise with the best available external clinical evidence that comes from systematic medical research, EBHI aims at integrating individual IT expertise with best available external evidence that comes from systematic health informatics research [16]. In the same year, two chapters on Evidence-Based Health Informatics appeared in a Health Informatics text book $[18,19]$. Also in 2010, IMIA included the term evidence-based health informatics in their recommendations on Education in Biomedical and Health Informatics [20], appreciating the need to teach basics of health IT evaluation and EBHI in health informatics curricula.

Thus the term Evidence-Based Health Informatics is now well over 10 years old, having been introduced in the context of evidence-based medicine. It became the focus of concerted effort in 2003, and has been refined in the context of evidence 
generation through evaluation by the IMIA and EFMI WGs. Since then it has been increasingly adopted in the health informatics community as a highly desirable principle, as its selection as the theme of this 2013 IMIA Yearbook of Medical Informatics a decade later demonstrates.

\subsection{Recommendations on Evidence-based Health Informatics}

A pivotal step in EBHI development arose from the aforementioned Declaration of Innsbruck in 2003, which in its twelve Recommendations included five methodological actions that form the practical basis of an evidence-based approach in health informatics [14]. These five methodological actions have been followed up by the IMIA and EFMI WGs and their international communities, namely:

- Guidelines for good evaluation practice should be made available": For EBHI, high-quality studies are needed. Recommendations can help here to establish a good evaluation practice. In the meantime, such guidelines are available as described in section 3.1.

- Terms, concepts and guidelines for reporting on results of ICT assessment studies should be made available": Complete and accurate publications of health IT evaluation studies are needed to establish a basis for EBHI. In the meantime, such guidelines are available - see section 3.2.

- "Evaluation networks should be established": Workshops and other activities at Medinfo and MIE conferences have been a first start, now being furthered by planned liaison between the relevant IMIA, EFMI, and AMIA groups. The EFMI-linked web site and mailing list at http://iig.umit.at/efmi/ is another contribution.

- "Appreciation of methods of evaluation should be part of health informatics curricula": As stated before, in the meantime, health IT evaluation and EBHI are part of the IMIA recommendations of health informatics education [20] see section 3.3.

- "An open access repository about evaluation studies should be established": Such a repository should contain information on planned, active and finalized evaluation studies. In the meantime, a repository of published health IT evaluation studies is available (see section 3.4).

Thus from the Innsbruck meeting and its resultant Declaration there was prioritised the need for well-designed and published evaluation studies, with the necessary methodology promoted by evaluation centres and evaluation networks and included in health IT curricula. In the decade since 2003, several significant activities and results have been achieved, to make possible obtaining the right information to make "a conscientious, explicit, and judicious use of current best evidence in making the decision of introducing and using IT in any given health care setting" [16].

These key outcomes are:

- Statement of Reporting Evaluations of Health Informatics Systems (STARE$\mathrm{HI}$ ), endorsed by the EFMI Board, adopted by the IMIA Council as an official IMIA document, and listed on the EQUATOR consortium website [21,22].

- Guideline for Good Evaluation Practice for Health Informatics Systems (GEP-HI) published to aid design and execution of evaluation studies and thus production of robust evidence [23].

- An abridged variant of STARE-HI for conference papers [24].

- A repository of over 1,700 references to published evaluation studies and reviews, located at http://evaldb.umit.at [25,26].

- Ongoing action plans to address emergent special application areas such as telemedicine [27].

The main sections of this paper will report on key methodological developments and frameworks in health informatics evaluation as the means of generating scientific evidence. Broadly speaking, these are seen as having the same value and significance as protocols and reporting standards used in clinical trials, and the analogy is important as health informatics systems directly or indirectly impact on clinical care delivery and therefore patients' well-being, and thus should aspire to the same levels of evidence robustness.

\section{Methods}

The authors are to a large degree the initiators and coordinators of most of the IMIA and EFMI initiatives in evaluation in the last decade, and are active in liaison with initiatives on a wider scale. To create an overview on current progress towards recognising and enabling EBHI, we first analysed the results of the individual but mainly joint work of the last 10 years of the IMIA and EFMI WGs, which has largely been published on http:// iig.umit.at/efmi. In addition, other international activities, especially on other health IT evaluation guidelines, were selected based on their prominence and then analysed.

Second, in order to give an inclusive view, we drew on Melia's review of the evaluation frameworks that currently exist in the field of health IT [28]. This work deduced from the cited papers a set of key generic evaluation principles in health informatics covering not just the EFMI and IMIA work but also other contributions to the literature. This forms the basis of section 4 .

Third, to identify the extent and nature of systematic reviews and meta-analyses on health IT evaluation, a systematic search was undertaken within the PubMed database of peer reviewed publications on $18^{\text {th. }}$ September 2012. The search details are presented in section 5 together with the results.

\section{Methodological Actions from the EFMI and IMIA Evaluation WGs}

\subsection{Guidelines for Good Evaluation Practice}

The Good Evaluation Practice guideline for Health Informatics (GEP-HI) was developed to support evaluators, health care professionals, decision makers and other health IT stakeholders in a systematic approach to design and execution of evaluation studies [23]. The guideline identifies some sixty essential issues that need to be considered during different phases of an evaluation study. The issues identified are related to evaluation study phases: preliminary outline, 
study design, operationalization of methods, project planning, execution and completion of the study. The guideline can be seen as an iterative spiral where the issues are repeated in depth or breadth to achieve progress during all phases.

The preliminary planning phase is the strategic phase which focuses on the purpose of the planned study and on the first ideas on why, for whom and how the evaluation study should take place. Examples of issues to be covered in this phase are: evaluation questions, identification of study stakeholders, organizational and user contexts for the study and explorative activities to establish the feasibility and relevance of the study. In the study design phase, a detailed evaluation study project plan is developed. Issues to be defined include key evaluation questions, indicators, stakeholder analysis, outline of study methods, organizational and technical contexts for the study, participants and timelines of the evaluation project and risk analysis and quality management. The operationalization of the methods phase makes the methodological approach and methods concrete and compliant with the evaluation object, the organizational context and the information need. Specifically this phase covers issues like definition of the study type, the frame of reference and outcome measures and evaluation criteria and quality control on measurement data. The project planning phase focuses on developing an evaluation project plan and procedures for project management, risk assessment and quality control. The execution of the evaluation study is a phase when the planned study is accomplished, data are collected and observations interpreted. During this phase, continuous quality control of findings and observation of changes needs to be implemented. The final phase, completion of the evaluation study, focuses on reporting of the study following the STARE-HI statement on reporting [21], on accounting and archiving the evaluation study materials and results and on formal closure of the study.

The GEP-HI guideline was developed during the period 2004-2011 through a consensus-seeking process within the community of health informatics evaluation experts, following on from the Innsbruck workshop. The guideline development started with utilisation of existing knowledge, experience and literature on evaluation, guidelines development, methodologies, codes of ethics and good implementation practices. An initial list of important elements was drafted and at regular intervals the draft guideline was presented, or submitted for discussion and feedback, to an increasing list of evaluation experts through EFMI's Working Group EVAL-mailing list. The draft versions of the guideline were also presented in workshops during several European and Medinfo health informatics conferences between 2005 and 2010. During these workshops feedback was collected and a progressively revised version was developed by the core team in consensus discussions and working sessions.

The strength of the GEP-HI guideline is that it forces evaluators to go through a checklist of relevant issues while planning and executing a health informatics evaluation study. The GEP-HI guideline has been introduced in the IMIA Yearbook 2012 and applied already in many papers and publications, such as $[29,30,31,32,33]$. The systematic approach of GEP-HI will contribute to a scientific approach on evaluation and further to evidence-based health informatics.

\subsection{Standards for Reporting of Evaluation Studies}

During the Innsbruck workshop it was recognized that though performing more rigorous evaluation studies is essential, on its own it is not sufficient for building the necessary evidence base for Health Informatics: rigorous scientific reporting of the research design and findings is required. In the late 1990s and early years of the $21^{\text {st }}$ century, guidelines appeared for reporting of several kinds of medical studies. The principle underlying these guidelines was the proper reporting of particular kinds of studies like clinical trials (the CONSORT Statement) [34], Diagnostic Accuracy Studies (STARD) [35], and Systematic Reviews (QUORUM) [36]. It was seen as essential, if health informatics was to take its responsible and rightful place as a health science, that evaluation studies in the domain of Health Informatics should follow the style and rigour of reporting guidelines generally accepted in the medical field, but that some additional information should be made available as well to be able to fully appreciate the findings. Installing health informatics technology (HIT) in a clinical environment is not only a technical endeavour; it is a change process that also has an impact on work processes and could be accompanied by organizational changes as well. These aspects are factors that will have an impact on the outcome of any study.

On this basis the Statement on Reporting of Evaluation Studies in Health Informatics (STARE-HI) [21] was produced, and it has become a generally applicable guideline that addresses the issues to be addressed in a report of an evaluation study of health informatics. STARE-HI was drafted by a core team, and successive drafts have been presented in workshops at various Medinfo and EFMI conferences to elicit feedback and suggestions for improvement. Comments have also been asked for through the mailing list of the EFMI working group on Evaluation - as this is a list with participants not only from Europe but also from the Americas and the Asian-Pacific region, this ensures a global input. The first formal version of STARE-HI as published in 2009 [21], and this version has been endorsed as indicated earlier in section 1.3.

STARE-HI consists of fourteen distinct sections on which guidance is given. It follows other guidelines by suggesting the use of structured abstracts, a policy that is currently also followed by the major Medical Informatics Journals - a small study was able to demonstrate that structured abstracts do indeed provide more information about the study than unstructured abstracts [37].

STARE-HI advocates an extensive description of the study site: type of hospital, extent of usage of the HIT application (for instance, only a few (specified) departments, or widespread), the timing of study with respect to implementation of the application in clinical use, any special measures taken prior to, during or after the implementation, the prospective users of the application, and a range of other factors. STARE-HI also addresses the topic of biases. It encourages the authors to report any biases that might affect the outcome of the study, and the approach taken to deal with them. For the results section, STARE-HI indicates the importance not merely of presenting the results, but also of reporting any unexpected events that may have influenced the findings, 
as well as unanticipated observations such as a usage that is different from the intended use of the application. Recognising the importance of conference presentations, but with their space constraint, a further piece of work has been to suggest how to prioritise the selection of STARE-HI reporting items in short papers [38].

Although there are some general guidelines for writing the discussion section of a study, many papers in Health Informatics are often weak in this area. Main findings are frequently not properly discussed, biases are not addressed, methodological issues are seldom discussed and if they are discussed it is often only the strong points that are addressed. Furthermore, limitations of a study are not always properly discussed. For this reason, STARE-HI also gives explicit guidance for the discussion section, addressing these kinds of shortcomings.

A further serious concern with respect to evaluation studies of HIT applications is the potential bias that is introduced when the study is performed by those that developed or implemented the application in the clinical environment. There is also a risk of 'socially acceptable answers', in particular a bias created by seeking to avoid causing embarrassment to those who sponsored the implementation policy or those who were the clinical users. Although there may not be a financial conflict of interest, there is certainly a conflict of interest between the role of evaluator who wants to undertake as objective a study as possible and the role of developer who wants to see its system being appreciated, or between those who promoted a system and those who have to make it work. STARE-HI suggests that full disclosure in these respects is given.

\subsection{Training in Good Health IT Evaluation}

The IMIA and EFMI WGs have organized health IT evaluation tutorials and workshops at several international conferences, to promote the idea of Evidence-based Health Informatics and to train for a systematic approach. Among others, workshops on STARE-HI and GEP-HI were organized at every MIE and Medinfo conference since 2003.
In addition, to learn about health IT evaluation, several textbooks are available, for example:

- Brender J. Handbook of Evaluation Methods for Health Informatics. Academic Press: 2006 [39].

- Friedman CP, Wyatt J C. Evaluation Methods in Biomedical Informatics. New York: Springer; 2006 [40].

The IMIA Recommendations on Education in Biomedical and Health Informatics [20] recognise the need to teach basics of health IT evaluation and EBHI both for IT users and for health informatics specialists. In particular, these recommendations include the following learning outcomes directly related to evaluation $[20$, table 2 ):

- "1.19 Evaluation and assessment of information systems, including study design, selection and triangulation of (quantitative and qualitative) methods, outcome and impact evaluation, economic evaluation, unintended consequences, systematic reviews and meta-analysis, evidence-based health informatics"

- "3.14 Usability engineering, human-computer interaction, usability evaluation, cognitive aspects of information processing" (see section 3.6)

Education in evaluation is vital for users, health informaticians, and health managers and policy makers involved in any aspect, if Evidence-based Health Informatics is to be appreciated and indeed recognised as a necessity and a policy and practice reality. The above examples, and the IMIA endorsement, show the importance, and the availability of material, but much remains to get this bedded into standard curricula.

Recognition of the importance of including evaluation in informatics curricula has also come from the United Sates, where (as well as important input to the IMIA Recommendations), there are two influential initiatives. In 2004 the American College of Medical Informatics produced a report on "Training of the next generation of informaticians", which included evaluation methods among the core skills necessary for informaticians and thus for inclusion in informatics curricula [41], while in 2009 evaluation of health information technology systems was also set as a core competency of the newly implemented U.S. clinical informatics medical subspecialty [42].

\subsection{Inventory of Health IT Evaluation Studies}

The IMIA and EFMI WGs provide and maintain the so-called Inventory of Health IT Evaluation Studies and Systematic Reviews, available for free at: http://evaldb.umit.at. This repository was created to help researchers to identify studies that have been conducted in defined settings. It now contains more than 1 700 references to published evaluation studies and reviews of evaluation studies of health information systems. A health information system in this context comprises all computer-based components that are used to enter, store, process, communicate, and present health related or patient related information, and which are used by health care professionals or the patient themselves in the context of inpatient or outpatient patient care. Each entry is indexed according to, among others, type of evaluated information system (e.g. telemedicine system, laboratory information system), clinical domain, evaluation method, and evaluation criteria. All entries are searchable based on a combination of parameters. For each entry, the abstract can be reviewed, and a direct link to PubMed is provided. The repository was initially based on a systematic literature search in PubMed conducted in 2003, and updated in 2006, 2009 and 2012. Since 2009, the repository is also updated based on input from researchers from all over the world. To support this, the repository website offers the possibility to propose studies that should be entered into the database.

Since 2010, between 150 and 200 individuals visit http://evaldb.umit at each month. Around $80 \%$ of the visitors use a direct bookmark to get to the site and around $10 \%$ are referred to it from other websites. This repository contributes to Evidence-based Health Informatics as it supports easy search and access to available evidence from health IT evaluation studies. It allows much more specific searches than, for example, the MeSH-terms in PubMed, and demonstrates the availability and feasibility of the scientific evidence base necessary for (and indicative of) a health science. 
Besides this repository, the EFMI working group also maintains a bibliography on evaluation papers and books (http://iig. umit.at/efmi/). Recently the EFMI, IMIA and AMIA working groups have joined forces to maintain this resource. A group of volunteers from around the world has been formed to regularly add relevant books, guidelines and papers on evaluation methodologies and methods, and also exemplary papers demonstrating outstanding study design and reporting.

\subsection{Meta-analysis in Health Informatics}

In order to progress the stated objective of enabling ,... evidence-based medicine, to make 'a conscientious, explicit, and judicious use of current best evidence' in making the decision of introducing and using IT in any given health care setting", [16] the right decision-making material is needed. Then come the questions "what will it require to make meta-analyses for evaluation studies?" and "what is the decision about?".

Meta-analyses and systematic reviews provide answers to different questions, or answers with different levels of certainty attached. The former has a quantitative nature while the latter has a qualitative (or quasi-quantitative) nature. When the question is like "Will CPOE reduce patients' risks of adverse events resulting from medication errors?" then a meta-analysis would be appropriate, while a question that includes investigation of functional impact or satisfaction may benefit with a richer answer from a systematic review.

What will it require to make meta-analyses of evaluation studies? The short answer is: 1) comparability, and 2) insight into the methodological set-up that enables inclusion and exclusion of studies or parts thereof. Following GEP-HI and STARE-HI stringently - or just STARE-HI - will to a large extent pave the way and facilitate meta-analyses through enabling an informed decision whether or not to include a study. It will also enhance the understanding of comparability among studies.

Key information to secure comparability and to enable the merge of data required for statistical pooling and analysis of data are: case characteristics, system characteristics and user characteristics. For instance, depending on the question addressed one may be unable to pool data from a community clinic with those of a university clinic; or one may be unable to merge performance data for a chief physician with a registrar or a nurse. It depends on the question addressed and the type of case organisation and system. Comparability is not only limited to the context of implementation (the organisation) and user characteristics. In Health Informatics, information systems are a key independent variable, impacts of which are evaluated.

Key information to secure insight facilitating inclusion and exclusion criteria for studies, even when all other parameters are comparable, is concerned with the methodological set-up. This all roots in the risk of bias; see these in [39]. Meta-analyses may become skewed in the event that the involved cases suffer from systematic bias. For example, a single candidate case may suffer from carry-over-effects caused by the study set-up; and another may suffer from extra-workload when there is no compensation of time invested by clinical staff in the evaluation; and yet another may suffer from the 'moving target' problem caused by the level of system maturity (e.g. technical maturity, organisational maturity such as in terms of time, of clinical operation, or user courses).

The obstacles for meta-analyses within health informatics are: a) low number of candidate systems for a given system type like CPOEs (for ordering laboratory investigations and/or for clinical medication handling), b) low number of comparable cases, and c) the cultural variety across countries, medical specialties and even from hospital to hospital. In pharmaceutical trials, the prescribed procedural usage of a drug may to some extent be specified by the pharmaceutical company, and anyway has to adhere to the clinic's normal procedures. In contrast, an IT-based system is deeply integrated into and interferes with the entire organisational set-up, down to minute aspects of the functions and responsibilities. The comparability may be alike for meta-analyses and systematic reviews, but it is easier verbally to moderate a statement for a qualitative analysis than to summarise such caveats in a statistical analysis or its graphical presentation. As for other evaluation questions, qualitative studies may in many respects replace - and answer - a quantitative study question and they are definitely less demanding. So, systematic reviews constitute a reasonable approach where meta-analysis is not achievable for one or another reason.

\subsection{Usability of Health Informatics Applications}

Usability is a fundamental dimension (attribute) of HIT products and systems that profoundly influences the impact of these products when they are put into use in clinical settings. Poor usability of HIT leads to "technology-induced errors" $[43,44]$, also referred to as "use errors" $[45,46]$ that compromise patient safety. It also diminishes the efficiency of the systems because they are difficult to use and difficult to learn how to use. Usability flaws of the systems are therefore one of the possible root causes for poor or negative impact of HIT.

For the last decade, the IMIA Working Group on Human Factors Engineering for Healthcare Informatics (HFE-HI) and the EFMI Working Group on Human and Organisational Factors of Medical Informatics (HOFMI) have collaborated with IMIA WG on Health Technology and Quality Assessment and EFMI WG on Assessment of Health Information Systems in their pursuit of evidence for Health informatics. Usability evaluations of HIT are part of the Human Factors Engineering (HFE) process [47,48], and are usually applied in the context of a Human Centred Design approach to HIT systems. Most usability evaluations aim at finding and fixing usability problems, and are therefore of formative nature.

The benefit of HFE methods to identify and prevent technology-induced errors is progressively being established [49,50]. As a consequence, a number of national and international initiatives and regulations for patient safety call for usability evaluation of HIT systems, especially those that would qualify as Medical Devices [50,51].

Seeking evidence for usability evaluation of HIT requires going one step further. As 
illustrated in figure 1 (retrieved from [52]), it is necessary to scientifically establish the relationship between (1) usability/design principles of HIT applications; (2) usability flaws of the developed systems, which are assumed to result of violations of usability principles; (3) usage problems experienced by the users as a consequence of usability flaws; and (4) negative outcomes such as use errors or poor clinical performance resulting from the usage problems.

Proper evaluations and rigorous reports of evaluations of HIT are necessary to track back negative impact or outcomes of HIT to root cause usability flaws and violations of usability principles. Evaluations of impact unfortunately rarely incorporate sufficient qualitative analysis to allow inferring usage problems and properly characterizing them. Specific usability evaluations are necessary to link identified usage problems with particular usability flaws. However scientific reports of usability evaluations suffer from recurrent weaknesses and inconsistencies making their systematic exploitation to support usability-related evidence difficult [53]. Therefore the IMIA and EFMI Human Factors WG support an ongoing initiative to elaborate a framework based on international consensus to guide scientific reports of usability studies of Health IT [50]. There are obviously strong relationships between this targeted specific framework and the existing STARE-HI guidelines [21].

\subsection{Indicators for Health Informatics Interventions}

In many sectors of health care and health systems, indicators increasingly are seen as a way of monitoring progress and highlighting problems needing further study or action. In health informatics and eHealth, well defined indicators that are based on compatible data provide a practical method to learn from eHealth initiatives and to monitor eHealth status and progress [54]. To this end, there is a rapidly growing interest in developing eHealth indicators. To make sense of an abundance of different proposed indicators, a conceptual framework for grouping the items is needed.

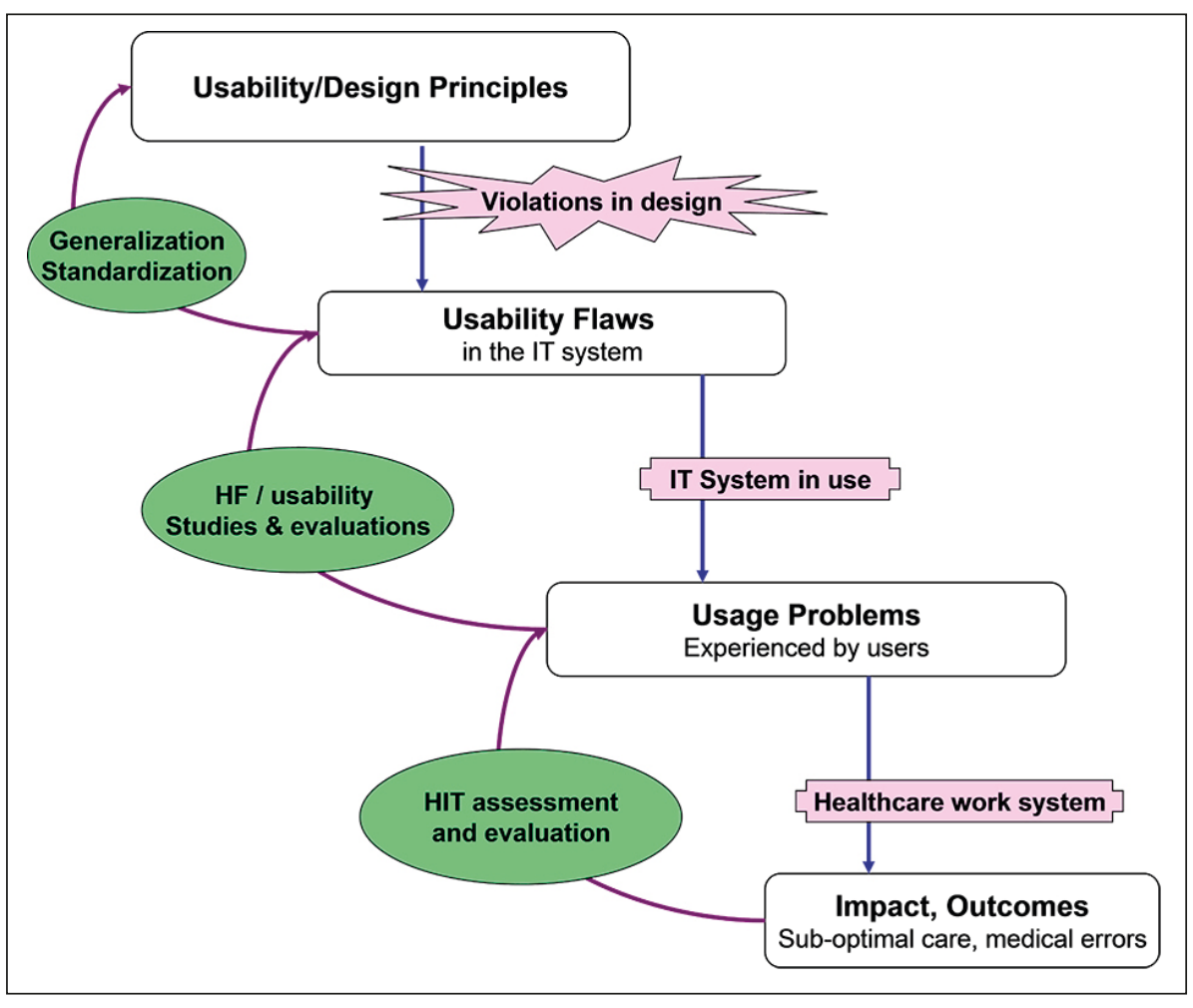

Fig. 1 Propagation of usability problems to healthcare outcomes (top-down process) and search for evidence to support usability principles (bottom-up process)

The inventory of evaluation studies of information technology in health care conducted by the IMIA and EFMI WGs [25] provides a good, robust framework for grouping of foci of Health IT evaluation studies, with structural information, process and outcome measures. Though indicator development is a separate subject of research, the indicator taxonomy should reflect and be compatible with the taxonomy of evaluation studies, since indicator development relies on existing evidence on impacts of particular systems or their functionalities.

For the basis of the indicator work, the taxonomy of evaluation studies was further mapped against the most commonly used frameworks for grouping eHealth indicators as part of an Indicator workshop at MIE 2012 supported by the EFMI evaluation working group [56]. This mapping has since been updated based on a systematic review of impacts of EHR content structures in different countries [56]. The frameworks that have been covered in the mapping include an Information Systems Success Model, covering elements of the system, information, system use and benefits [57] and the Health Technology Assessment (HTA) evaluation domains, where outcome-indicators are most prominent [58]. A systematic analysis of concepts in these different frameworks from different scientific traditions is still needed for a more detailed, scientifically grounded, conceptual framework for grouping of eHealth indicators.

Another basis for the eHealth indicator work was laid by defining a comprehensive methodology to develop eHealth indicators. It has been published as a joint paper with the IMIA and EFMI WGs [59]. It includes four main phases:

1) Defining the context (human and environmental) for measurement with two primary components: key stakeholders and the relevant area or system.

2) Defining the goals.

3) Defining methods for indicator selection and categorization. Grouping of different variables found in various studies is one important part of this step.

4) Defining the data. 
This methodology is compatible with general guidelines for health IT evaluation practice - the GEP-HI guidelines [23]. This is important, since the process of defining indicators, collecting data and reporting results (indicator-based monitoring of eHealth progress) bears great resemblance to conducting an evaluation study.

This indicator methodology is currently being tested in the Nordic eHealth indicator work [60]. The importance of following through all four steps has been revealed in the methodology testing: one of the main outcomes has been specification of the systems/ services/ functionalities for which indicators are developed, in order to provide comparable data of availability, use and impacts of these functionalities in different countries. Analysis of the Nordic eHealth policies has proven necessary to define common goals for which common indicators are developed, and linking indicator data to policy development. For this phase, a framework for grouping of indicators is essential. Testing of data collection has proven the importance of reliable and valid data, and challenges created by different formulations of the variables.

Indicator-based monitoring of eHealth progress generates one line of evidence towards EBHI, supported by more detailed evaluation studies. The decade of work and achievements by the eHealth evaluation community has been essential for the development of common eHealth indicators to be used by eHealth decision makers, developers and users. It is necessary to draw on these results also in the future indicator work to maintain a coherent evidence-base.

\section{Other International Activities}

Though the work undertaken by the IMIA and EFMI WGs working in harmony has been coordinated and significant, it is not the only evaluation initiative which has produced important conceptual frameworks, as has been described in two earlier editions of this Yearbook [61,2] and in a more recent analysis [28]. The following initiatives and concepts on HIT evaluation, either on developing, designing or reporting evaluation studies, can be distinguished.

\section{Human, Organisational and Technology HOT-fit}

Yusof et al. [62] devised an evaluation framework which considers human, organisation and technology (HOT-fit) factors. The framework recognises interrelated dimensions of health informatics systems (HIS) success which influence each other and determine both benefits and satisfaction. Quality of aspects has a causal and well as outcome position. 'Fit' concerns the ability of HIS, stakeholders, and clinical practices to align with each other. However, in practice the classification of evaluation data reportedly affected the flow of the narrative of results, which suggests limitations to application in practice of the HOT-fit framework, which is further compounded by the fact that "the concept of fit is perceived as complex, abstract and subjective" [62]. This framework can be applied together with the GEP-HI guideline as it provides support for methodical choices within the overall design and execution of an evaluation study [GEP-HI].

\section{AHRQ Evaluation Toolkit}

For the United States Agency for Health Research and Quality (AHRQ)'s National Resource Center, an evaluation toolkit was developed to provide step-by-step guidance for developing evaluation plans for health information technology projects [63]. The toolkit assists evaluators to define the goals for evaluation, what is important to stakeholders, what needs to be measured to satisfy stakeholders, what is realistic and feasible to measure, and how to measure these items. Examples are presented with suggested evaluation methodologies for each. The toolkit is very useful from the methodological point of view, but it does not give guidelines for the evaluation project itself, how to manage it, how to carry out the project, or how to complete and report the study, and thus details only part of the GEP-HI coverage. Indeed, the two approaches should be seen as complementary.

\section{Model for Assesment of Telemedicine (MAST)}

The Model for ASsesment of Telemedicine (MAST) framework is the result of a European Commission project, and was designed to be used where the purpose is to describe effectiveness and contribution to quality of care of telemedicine applications, and to produce a basis for decision making. Kidholm et al. suggest that "the aim is that clinical, administrative and political decision makers in hospital, communities, regions, government departments will use the model as a structure for the description of the outcomes of telemedicine and as an important basis for decisions on whether or not to implement telemedicine services in health care systems" [64].

MAST starts with a preceding analysis, then assesses the application against seven categories: Health Problem and characteristics of the application; Safety; Clinical effectiveness; Patient perspectives; Economic aspects; Organisational aspects; and Socio-cultural, ethical, legal aspects. Finally MAST seeks an assessment of the transferability of results. MAST does not consider the execution and management of an evaluation project, and focuses solely on telemedicine systems rather than health IT generally, and has only been devised in the context of local inter-active telemedicine applications. However, as it follows a HTA format, the process is familiar to stakeholders in the EU and to the health care industry.

\section{Socio-Technical Approach - STAT-HI}

Recognition that health informatics and e-health systems bring ICT into a complex health system of socio-dynamics rather than a static data setting has been emphasised by several analysts including Lorenzi et al. [65], Berg with several collaborators $[66,67,68]$, and Ash et al. such as in [69]. To address this, a Socio-technical Assessment Tool STAT$\mathrm{HI}$ has been postulated [33]. This claims compatibility with GEP-HI and STARE-HI, while focussing on the socio-technical aspects of systems and their implementation in the health care organisational environment. 


\section{Other Reporting Guidelines}

Since the publication of STARE-HI, other reporting guidelines for specific types of evaluation studies or applications have been published. This includes, on the methodological side, the reporting of qualitative studies [70], and on applications side, the reporting of evaluation studies on health social media and $\mathrm{m}$-health [71], and of a postulated framework for telemedicine evaluation [72]. Such specialized reporting guidelines are useful since they give additional guidance for specific kind of studies, yet without cross-linkage they have a potential to lead to fragmentation of the potential methods and evidence-bases. We expect more such guidelines to appear, for example for HFE evaluation studies, but hope for greater sector cohesion within these. This should result in a series of related guidelines for evaluation studies in health informatics which, when applied rigorously, will further facilitate systematic reviews and meta-analyses to build up the evidence base of health informatics.

\section{Bellagio e-Health Evaluation}

A new top-level objective for e-health evaluation has also been published in the form of the World Health Organisation's Call to Action on Global eHealth Evaluation [73], primarily in the context of e-health systems for developing countries. This comprises nine high-level principles, which include an evidence-based culture, high quality evaluative data collection, and stakeholders engagement.

These principles emphasise the overall responsibility to ensure that eHealth solutions and policies are subjected to, and informed by, rigorous evaluations and that evaluation findings should be used and contribute to evidence generation, synthesis and documentation, including peer-reviewed articles [73]. The Bellagio principles do not guide how to plan, execute and report evaluation studies, for which methods such as GEP-HI and STARE-HI apply.

\section{Ten Consistent Principles}

Thus there has been considerable other work additional to the GEP-HI and STARE-HI products of the IMIA and EFMI groups, addressing specific contexts or aspects. Melia has analysed from a literature review of the major ones up to 2011 that ten core principles are constant from these as essentials for effectiveness in all evaluations [28]:

- Preliminary Planning

- Stakeholder Analysis

- Health issue and E-health application

- Safety

- Clinical Effectiveness

- User Experience

- Economic Aspects

- Organisational Aspects

- Ethical and Legal Issues

- Reporting

Table 1 Structured Queries for PubMed Search for Health Informatics Evaluations

\begin{tabular}{|c|c|}
\hline Query 1: () & Query 2: \\
\hline 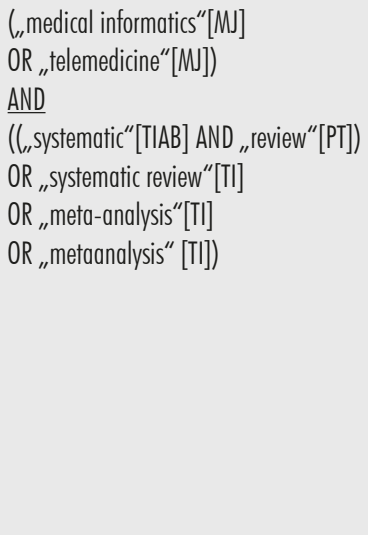 & 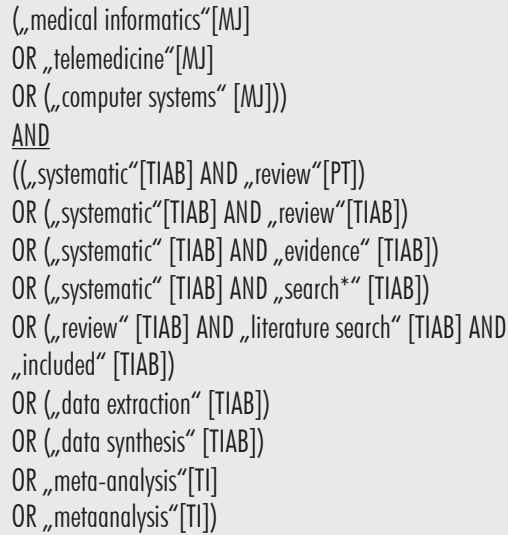 \\
\hline
\end{tabular}

\section{A Structured PubMed Search on Published Systematic Reviews}

The PubMed search previously referred to in the Methods section comprised two queries (see Table 1). The results of both queries were combined and duplicates were eliminated.

The titles and abstracts of the retrieved papers were then read by two researchers to identify systematic reviews on health IT. Inclusion and exclusion criteria were discussed and decided jointly by both researchers. Table 2 shows the inclusion and exclusion criteria used.
Table 2 Inclusion and Exclusion Criteria for Search for Health Informatics Evaluations

\begin{tabular}{|l|l|}
\hline Inclusion criteria: & Exclusion criteria: \\
\hline - Clear indication of systematic review (not & - Reviews on evaluation methodologies \\
narrative review, overview or opinion paper) & - Reviews on quality of evaluation studies \\
- Focus on health IT (e.g. clinical information & - Reviews on software products on the market \\
systems, telemedical applications, patient & - Reviews on image and signal processing systems \\
information systems) & - Reviews on bioinformatics applications \\
- Review addresses adoption, quality or impact & - Reviews on robotics or computer-aided navigation \\
of a health IT system & - Reviews on virtual reality systems \\
- IT is used in practice (not only in research) & - Reviews on e-learning systems for clinical staff \\
& - Reviews on diagnostic tests \\
& - Reviews on lifestyle applications (e.g. web site for \\
& - weight, alcohol, smoking cessation) \\
- Reviews on body-worn sensors \\
- Reviews on data quality in databases \\
- Reviews on quality of health web sites \\
- Reviews on quality of algorithms \\
\hline
\end{tabular}

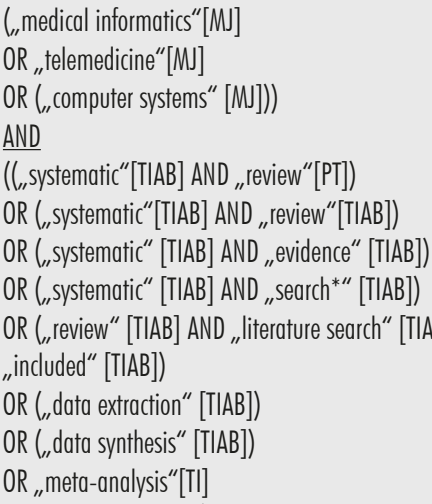


As described in section 2, a systematic PubMed query was conducted to identify system reviews on health IT. Query 1 retrieved 1,032 papers, while query 2 retrieved 2,124 papers. Once duplicates were eliminated 1,092 unique references were found. Reading of title and abstract identified 236 reviews that met the inclusion and exclusion criteria and were thus identified as systematic reviews on health IT.

Figure 2 presents the number of systematic reviews published each year, in relation to all published papers with "medical informatics" as major MeSH Heading.

This shows a steady growth in health informatics papers, but a surprising and sustained tailing off from 2008, even though interest in informatics applications has continued to develop, technological and service opportunities have grown, and e-health policies have strengthened in an increasing number of countries. The lag behind in systematic reviews is to be expected to reflect the buildup of literature. The continued growth with no sustained drop-off can be interpreted as fuelled by two factors - increased but more sophisticated policy interest, and strengthened desire to make best use of available evidence. What this simple study cannot determine is the quality of the published evaluations used in the 236 systematic reviews, nor the quality of the systematic reviews or the proportion that performs robust meta-analyses. Repeating the search on other bibliographic repositories might have yielded additional studies, but was not possible within the resources available.

\section{Discussion}

The Introduction to this paper emphasised the importance of Evidence-based Health Informatics and the need for a culture change towards it becoming the universal yardstick, while subsequent sections outline the current state of the art. These are still early days, with much ground to be covered. It is therefore important to remain within the current 'art of the possible', and aim for steady progress in applying policy and practical tests; this means not making hurdles so high they are impossible, nor so low they let inappropriate policies and products through.

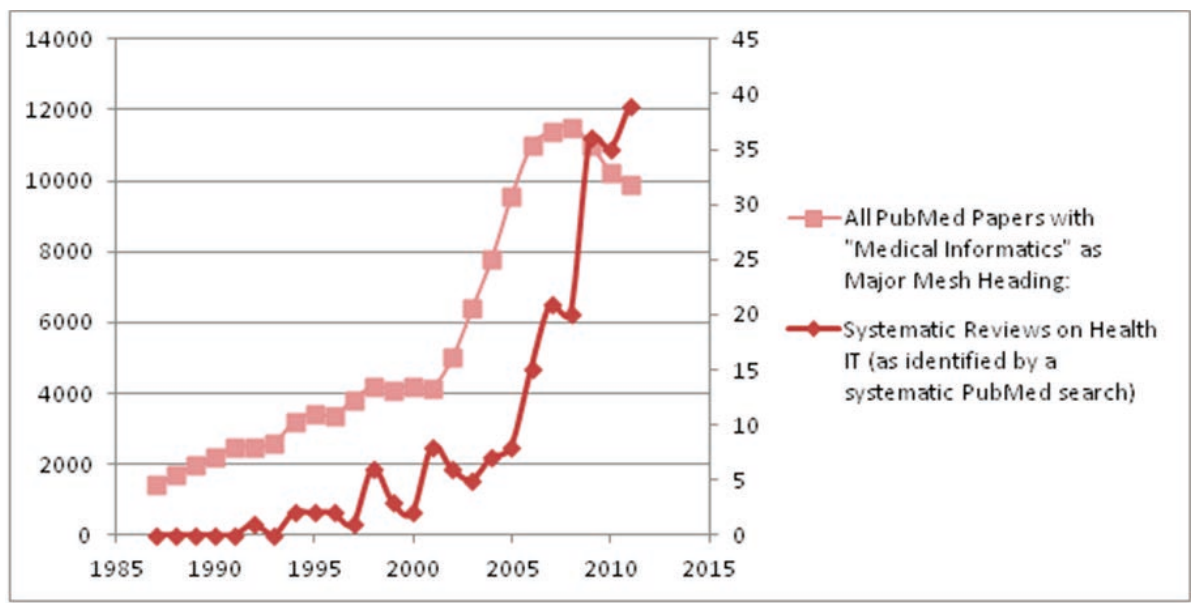

Fig. 2 Numeric Results of PubMed Search for Health Informatics Evaluations

This paper has focussed primarily on the work undertaken by the IMIA and EFMI working groups, and this has been concerted action enriched by regular iterations at international health informatics conferences. We have also reported on some of the stronger items in the wider literature on methods, the issues of particular application areas, and emergent issues. This has shown the strength of interest in this important field, but also the degree of fragmentation, failure to cross-reference published material and indeed recognised principles, or linkage to established evaluation and reference frameworks.

This report is inevitably a somewhat limited and biased snapshot. There is almost certainly other important evaluation activity, which is not reported, or which has not come up in our searches (which are largely restricted to English language sources). Similarly, some publications in the policy, economic and sociological literature may have eluded us. Finally, many evaluations (of various scientific rigour according to the standards promoted here) and other literature may remain within the grey literature, and in internal organisational and vendor settings. But in turn, this limitation highlights the importance of a more mature sharing of knowledge - including, importantly, learning from adverse or disappointing outcomes - so as to further the maturity and richness of the science of EBHI. This is necessary since to enable operational level and indeed national policy makers to more easily and confidently access and interpret the literature without ambiguity or uncertainty, greater cohesion is needed.

The forthcoming anticipated collaboration between WHO and IMIA [1], and the move to stronger links between work in IMIA, EFMI, and the American Medical Informatics Association (AMIA) through their working groups on evaluation of health informatics applications, are very significant and welcome next steps. At the same time, the lack of engagement with the policy making community, particularly at national level, is of great concern, and hopefully the WHO initiatives will blaze a trail in this direction. Another difficulty is that of obtaining funding for evaluation or for systematic reviews, with an over-riding emphasis on building and installing systems rather than learning from experience as to how to design and implement health informatics systems more effectively and safely.

Meanwhile, the barriers to evaluation and to an evidence-based approach must be understood if they are to be addressed. They include cost and opportunity cost, organisations' and professionals' sensitivities about their practice, policy makers' concerns about exposing their HIT investment decisions (which may have been forward-looking and courageous), and practical and technical difficulties [13]. However, the difficulties are being overcome and the maturity of experience should ensure a demand for widespread application, while the volume of material is becoming sufficient for meta-analyses to become feasible in some fields. 


\section{Applying an Evidence-based Approach in Health Informatics Policy}

The creation and application of an evidence-based approach is part of a continuum. Policy, including organisational investment plans, should be evidence-based, but that requires access to relevant evidence which until now has been difficult due to lack of systematic creation and availability of impartial evidence. However, even when the evidence does become available, that does not guarantee that it will result in effective use. As has been put forward in an earlier IMIA Yearbook, the creation of evidence-based policy in any field in healthcare is far more elusive than it ought to be [2]. It was in 1995 that an editorial in the British Medical Journal asked: “... at a time when ministers are arguing that medicine should be evidence based, is it not reasonable to suggest that this should also apply to health policy?'[74]. In health policy generally this concept has only moved forward slowly. A modest but sound literature on this has built up; see for instance $[75,76]$, but far from all health policies are evidence-based. Regrettably this is particularly the case in health informatics (or e-Health) policy, where belief, aspiration, and the apparent need to be seen to be progressive seem equally influential. Similarly, there is limited objectivity in many investment decisions, where vendor promises of products yet to be finalised, and imperfect fits to imperfect tender specifications, often prevail.

Hitherto the lack of scientific evidence to support evidence-based policy in health informatics has been a reasonable excuse, but these days should now be ending, while investment in evidence generation through evaluation should be seen as cost-effective. However, with the increasing availability of evidence and the means of obtaining it, whenever health informatics investment is proposed (not least in the context of policies towards 'e-health'), it should be considered irresponsible to proceed without evidence that the new approach will operate without failure or unanticipated adverse effects. And as has been shown, detrimental effects up to and including patient deaths can occur [7], as can organisational inefficiencies, and staff having to use 'work-arounds' to avoid functional problems. Yet, unlike patients or clinicians, those who promote or implement new health informatics policies put neither their lives nor their livelihoods directly at risk, so they should be expected to make evidence-based policy and investment decisions as part of ethical decision-making.

\section{Future Needs and Trends in Health Informatics Evaluation and Evidence}

However, health informatics systems and applications are not static, and neither is the underpinning technical and software engineering science. So, further work on evidence creation continues to be necessary.

On the one hand, this relates to the need to consider new application areas, such as new web technologies, social media, integrated monitoring and cross-border and fully virtual care $[2,27]$. The second area involves the need to increase the understanding in management and policy circles, not least because of the emergent and welcome focus on policies to create smarter ICT-reliant health system [77]. Thirdly, there needs to be further discussion on regulatory and control mechanisms, weight of evidence (possibly related to seriousness of risk, as with the CE-marking system), and related mechanisms such as trusted third party incident reporting mechanisms. Finally, societal (and professional) expectations of health care systems overall are rising in the light of increased consumer awareness, needing development of evaluation methods and criteria, not least in usability and in governance.

The current mechanisms within IMIA and its members and regional groups, and the current WHO initiatives, can form important springboards for this. But greater openness and discussion, within health informatics, and then with the policy and patient communities, are needed.

\section{Conclusion}

The central importance of health informatics as a tool - indeed a unifying mechanism within health care is now globally self-evident. Health informatics is science-based, yet hitherto it has afforded itself (and has been allowed) the luxury of not having to apply the rigorous scrutiny required for other health sciences. This now has to stop, and GEP-HI and STARE-HI in particular provide the guidelines for a first step.

Health informatics applications can be, and usually are, beneficial. Often, they could be more beneficial if they were better understood at the application level and location. But on occasions, health informatics can be, and has been shown to be, wasteful, harmful, and even fatal [7].

This luxury of low accountability, and excessive trust in promises, is outmoded and dangerous; it is also damaging credibility and thus jeopardising the potential benefits. It portrays health informatics as an immature discipline, not yet being a full health science, and as not applying either health or business ethics. This must cease. Evidence-based Health Informatics is feasible and achievable, and should become the norm and the expectation. The methods are here, and need promoting along with a continuing development programme to keep pace with application development and rightful societal expectations.

\section{Acknowledgments}

It is important to acknowledge the essential contributions of all participants in many workshops, tutorials and seminars as well as members of the virtual health informatics evaluation community for their contribution and feedback, in not only the IMIA and EFMI led work, but also the other studies. These have been instrumental in contributing to the development of models and of evaluations which form the emergent knowledge base.

\section{References}

1. http://www.imia-medinfo.org/new2/GA/2012beijing/1210GA-beijing-president-WHO.pdf (accessed 31 January 2013).

2. Rigby M, Ammenwerth E, Talmon J, Nykänen P, Brender J, de Keizer N. Health Informatics 3.0 and other Increasingly Dispersed Technologies Require Even Greater Trust: Promoting Safe Evidence-based Health Informatics. In: Geissbuhler A, Kulikowski CA, editors. IMIA Yearbook of Medical Informatics 2011: Towards Health Informatics 3.0. Stuttgart: Schattauer; 2011. p. 105-11. 
3. Beauchamp T, Childress JF. Principles of Biomedical Ethics. 6th ed. New York: Oxford University Press; 2009.

4. European Commission. Communication from the Commission on the Precautionary Principle, $\operatorname{COM}(2000)$ 1, Brussels; 2000.

5. Fisher E, Jones J, von Schomberg R, editors. Implementing the Precautionary Principle: Perspectives and Prospects. Cheltenham, UK and Northampton, MA, US: Edward Elgar; 2006.

6. Ammenwerth E, Shaw NT. Bad health informatics can kill - is evaluation the answer? Methods Inf Med 2005;44(1):1-3.

7. Web list "Bad Health Informatics can Kill" (accessible via the Bad Health Informatics link at http:// iig.umit.at/efmi) (accessed 31 January 2013).

8 http://business-ethics.com/ (accessed 31 January 2013).

9. http://www.ibe.org.uk/ (accessed 31 January 2011)

10. Patrick, JD. The Validity of Personal Experiences in Evaluating HIT. Appl Clin Inf 2010;1:462-5.

11. http://eur-lex.europa.eu/LexUriServ/LexUriServ. do? (accessed 31 January 2013)

12. Haynes RB, Hayward RS, Jadad AR, Sebaldt RJ. Evidence based health informatics: an overview of the Health Information Research Unit at McMaster University. Leadersh Health Serv 1996 May-Jun;5(3):41-4.

13. Rigby M, Evaluation: 16 powerful reasons why not to do it - and 6 over-riding imperatives. In: Patel V, Rogers R, Haux R, editors. Proceedings of the 10th World Congress on Medical Informatics (Medinfo 2001). Amsterdam: IOS Press; 2001. p. 1198-202.

14. Ammenwerth E, Brender J, Nykänen P, Prokosch H-U, Rigby M, Talmon J, et al. Visions and strategies to improve evaluation of health information systems. Reflections and lessons based on the HIS-EVAL workshop in Innsbruck. Int J Med Inf 2004 Jun 30;73(6):479-91.

15. Ammenwerth E. Health technology assessment. Findings from the Section on Assessing Information Technologies for Health. Yearb Med Inform 2006:16-9.

16. Ammenwerth E, Schnell-Inderst P, Siebert U. Vision and Challenges of Evidence-Based Health Informatics: A case study of a CPOE meta-analysis. Int J Med Inform 2010; 79: e83-e88.

17. Sackett D, Rosenberg W, Gray J, Haynes R, Richardson S. Evidence based medicine: what it is and what it isn't, BMJ 1996;312(7023):71-2

18. Ammenwerth E. Evidence based health informatics. Stud Health Technol Inform 2010;151:427-34

19. Wyatt J. Assessing and improving evidence based health informatics research. Stud Health Technol Inform 2010;151:435-45.

20. Mantas J, Ammenwerth E, Demiris G, Hasman A, Haux R, Hersh W, et al. Recommendations of the International Medical Informatics Association (IMIA) on Education in Biomedical and Health Informatics. IMIA White Paper. Methods Inf Med 2010;49(2):1 - 16

21. Talmon J, Ammenwerth E, Brender J, de Keizer N, Nykänen P, Rigby M. STARE-HI - Statement on reporting of evaluation studies in Health Informatics. Int J Med Inform 2009; 78(1): 1-9.

22. http://www.equator-network.org/resource-centre/ library-of-health-research-reporting/reporting-guidelines/other-reporting-guidelines/ (accessed 31 January 2013).

23. Nykänen P, Brender J, Talmon J, de Keizer N, Rigby M, Beuscart-Zephir M-C, et al. Guideline for good evaluation practice in health informatics (GEP-HI). Int J Med Inform 2011;80:815-27.

24. de Keizer NF, Talmon J, Ammenwerth E, Brender J, Nykanen P, Rigby M. Mini Stare-HI: Guidelines for Reporting Health Informatics Evaluations in Conference Papers. In: Safran C, Reti S, Marin HF, editors. MEDINFO 2010 - Proceedings of the 13th World Congress on Medical Informatics. Amsterdam: IOS Press; 2010. p. 1206-10.

25. Ammenwerth E, de Keizer N. An inventory of evaluation studies of information technology in health care: Trends in evaluation research 1982 2002. Methods Inf Med 2005;44:44-56.

26. Ammenwerth E, de Keizer N. Evaluation Database - A web-based inventory of evaluation studies in medical informatics; Institute for Health Information Systems, University for Health Sciences, Medical Informatics and Technology, Austria (on-going) (available at http://evaldb.umit.at) (accessed 31 January 2013).

27. Rigby M, Brender J, Beuscart-Zephir M-C, Hypponen $\mathrm{H}$, Nykänen $\mathrm{P}$, Talmon J, et al. Next Steps in Evaluation and Evidence - from Generic to Context-Related. In: Moen A, Andersen SK, Aarts J, Hurlen P, editors. User Centred Networked Health Care-Proceedings of MIE 2011. IOS Press; 2011. p. 208-12.

28. Melia S. The challenge of evaluating telehealth interventions - Dissertation in part fulfilment of the award of MBA (Health Executive), Keele University, 2012.

29. Nykänen P, Kaipio J, Kuusisto A. Evaluation of the Finnish nursing classification system and four nursing documentation systems in Finland - lessons learned and directions or the future. Int J Med Inform 2012:81(8),505-20.

30. Kopanitsa G, Tsvetkova Z, Veseli H. Analysis of metrics for usability evaluation of electronic health record systems. In: Blobel B, editor. Large-scale projects in eHealth. Amsterdam: IOS Press; 2012. p. 129-33.

31. Anttila M. Adoption of information technology based patient education in psychiatric nursing. $\mathrm{PhD}$ dissertation, University of Turku, 2012. Accessible at: http://www.doria.fi/handle/10024/74662 (accessed 31 January 2013).

32. Oluoch T, Santas X, Kwaro D, Were M, Biondich $\mathrm{P}$, Bailey $\mathrm{C}$, et al. The effect of electronic medical record-based clinical decision support on HIV care in resource-constrained settings: a systematic review. Int J Med Inform 2012;81(10):e83-92.

33. Scott PJ, Briggs J. STAT-HI: A socio-technical assessment tool for health informatics implementations. Open Med Inform J 2010;4:214-20.

34. Moher D, Schulz KF, Altman DG. The CONSORT statement: revised recommendations for improving the quality of reports of parallel-group randomised trials. Lancet 2001 Apr 14; 357(9263):1191-4.

35. Bossuyt PM, Reitsma JB, Bruns DE, Gatsonis CA, Glasziou PP, Irwig LM, et al. Towards complete and accurate reporting of studies of diagnostic accuracy: the STARD initiative. Standards for Reporting of Diagnostic Accuracy. BMJ
2003;326(7379):41-4.

36. Moher D, Cook DJ, Eastwood S, Olkin I, Rennie D, Stroup DF. Improving the quality of reports of meta-analyses of randomised controlled trials: the QUOROM statement. Quality of Reporting of Meta-analyses. Lancet 1999 Nov 27;354(9193):1896-900.

37. Talmon J, Ammenwerth E, Geven T. The quality of reporting of health informatics evaluation studies: a pilot study. Stud Health Technol Inform 2007;129 (Pt 1):193-7.

38. de Keizer NF, Talmon J, Ammenwerth E, Brender J, Rigby M, Nykänen P. Systematic Prioritization of the STARE-HI Reporting Items. An Application to Short Conference Papers on Health Informatics Evaluation. Methods Inf Med 2011 Mar 4; 50(3).

39. Brender J. Handbook of Evaluation Methods for Health Informatics. New York: Academic Press; 2006. p. 361

40. Friedman CP, Wyatt JC. Evaluation Methods in Biomedical Informatics. New York: Springer; 2006.

41. Friedman CP, Altman RB, Kohane IS, McCormick KA, Miller PL, Ozbolt JG, et al. Training of the next generation of informaticians: the impact of "BISTI" and bioinformatics - a report of the American College of Medical Informatics. J Am Med Inform Assoc 2004;11(3):167-72.

42. Gardner RM, Overhage JM, Steen EB, Munger BS, Holmes JH, Williamson JJ, et al. Core Content for the Subspecialty of Clinical Informatics; J Am Med Inform Assoc 2009; 16:153-7.

43. Borycki E, Kushniruk A. Identifying and preventing technology-induced error using simulations: application of usability engineering techniques. Healthc Q 2005;8 Spec No:99-105.

44. Kushniruk AW, Triola MM, Borycki EM, Stein B, Kannry JL. Technology induced error and usability: the relationship between usability problems and prescription errors when using a handheld application. Int J Med Inform 2005 Aug;74(78):519-26.

45. International Electrotechnical Commission. Medical Electrical Equipment - General requirements for basic safety and essential performance. Collateral Standard. Usability. Geneva: International Electrotechnical Commission; 2007. Report No.: IEC 60601-1-6).

46. International Electrotechnical Commission. Medical Electrical Equipment - General requirements for basic safety and essential performance. Collateral Standard. Usability. Geneva: International Electrotechnical Commission; 2010. Report No.: IEC 60601-1-6.

47. Beuscart-Zephir MC, Elkin P, Pelayo S, Beuscart $R$. The human factors engineering approach to biomedical informatics projects: state of the art, results, benefits and challenges. Yearb Med Inform 2007;109-27.

48. Beuscart-Zephir MC, Pelayo S, Borycki E, Kushniruk A. Human Factors Considerations in Health IT Design and Development. In: Carayon P, editor. Human Factors and Ergonomics in Health Care and Patient Safety 2nd Edition. CRC Press; 2012. p. 649-70.

49. Borycki E, Kushniruk A. Where do technology-induced errors come from? Towards a model for conceptuallizing and diagnosing errors caused by 
technology. In: Kushniruk A, Borycki E, editors. Human, Social and Organizational Aspects of Health Information Systems. Hershey, NY, Idea Group; 2008.

50. Beuscart-Zephir MC, Borycki E, Carayon P, Jaspers MW, Pelayo S. Evolution of Human factors Research and Studies of Health information technologies: the Role of Patient safety. Yearb Med Inform 2013. Seitenzahlen

51. Magrabi F, Aarts J, Nohr C, Baker M, Harrison S, Pelayo S, et al. A comparative review of patient safety initiatives for national health information technology. Int J Med Inform 2013 May;82(5):e139-48.

52. Marcilly R, Beuscart-Zephir MC, Pelayo S. Seeking Evidence to support Usability Principles for medication-related CDS functions. 2013.

53. Peute LW, Spithoven R, Bakker PJ, Jaspers MW. Usability studies on interactive health information systems; where do we stand? Stud Health Technol Inform 2008;136:327-32.

54. Lawrence M, Olesen F. Indicators of Quality in Health Care. Eur J Gen Pract 1997;3(3):103-8.

55. Hyppönen H, Ammenwerth E, Faxwaag A, Nohr C, Walldius $\AA$. eHealth indicators: results of the MIE 2011 workshop. Stud Health Technol Inform 2012;180:328-32.

56. Hyppönen H, Vuokko R, Mäkelä-Bengs P, Doupi P, Saranto K, Mäkelä M. Impacts of structuring the patient record: a systematic review protocol and results of previous reviews. [Submitted to Int $\mathrm{J}$ Med Inform, under review]

57. DeLone WH, McLean ER. The DeLone and McLean Model of Information Systems Success: a ten-year update. Journal of Management Information Systems 2003;19(4):9-30.

58. Lampe K, Mäkelä M, editors. HTA Core Model for medical and surgical interventions. First Public Draft (Revised) EUnetHTA: Work Package 4, 2007, available at http://www.eunethta.eu/outputs/ hta-core-model-medical-and-surgical-interventions-10r (accessed 31 January 2013).

59. Hyppönen H, Ammenwerth E, de Keizer N. Exploring a methodology for eHealth indicator development. Stud Health Technol Inform 2012;180:338-42.
60. Hyppönen, H., Faxvaag A, Nøhr C, Vimarlund V, Hardardottir GA , Reponen J, et al. Nordic eHealth Indicators - Organisation of research, first results and plan for the future. [in press, Nordic Council of Ministers, Tema Nord-series number TN2013:522]

61. Rigby M. Evaluation - the Cinderella Science of ICT in Health. In Kulikowski C, Haux R, editors. IMIA Yearbook of Medical Informatics 2006. Stuttgart: Schattauer; 2006. p. 114-20.

62. Yusof MM, Kuljis J, Papazafeiropoulou A, Stergioulas LK. An evaluation framework for Health Information Systems: human, organization and technology-fit factors (HOT-fit). Int J Med Inform 2008 Jun;77(6):386-98.

63. Cusack CM, Byrne C, Hook JM, McGowan J, Poon EG, Zafar A. Health information technology evaluation toolkit: 2009 Update (Prepared for the AHRQ National Resource Center for Health Information Technology under Contract No. 29004-0016.) AHRQ publication No. 09-0083-EF. Rockville, MD: Agency for Healthcare Research and Quality. June 2009.

64. Kidholm K, Bowes A, Dyrehauge S, Granstrøm Ekeland A, Flottorp SA, Kvistgaard Jensen L, et al. The MAST Manual. MAST-Model for assessment of telemedicine. http://www.epractice.eu/en/ library/315610 (accessed 31 January 2013)

65. Lorenzi N, Riley R, Blyth A, Southon G, Dixon B. Antecedents of the people and organizational aspects of medical informatics. J Am Med Inform Assoc 1997;4(2):79.

66. Berg M. Medical work and the computer-based patient record: a sociological perspective. Methods Inf Med 1998; 7(3):294-301.

67. Goorman E, Berg M. Modelling nursing activities: electronic patient records and their discontents. Nurs Inq 2000;7(1):3-9.

68. Aarts J, Doorewaard H, Berg M. Understanding implementation: the case of a computerized physician order entry system in a large Dutch university medical center. J Am Med Inform Assoc 2004;11(3):207-16.

69. Ash JS, Sittig DF, Dykstra RH, Guappone K, Carpenter JD, Seshadri V. Categorizing the unintended sociotechnical consequences of computerized provider order entry. Int J Med Inform 2007;76(Suppl
1):21-7.

70. Niazkhani Z, Pirnejad H, Aarts J, Adams S, Bal R. Reporting qualitative research in health informatics: REQ-HI recommendations. Stud Health Technol Inform 2011;169:877-81.

71. Eysenbach G; CONSORT-EHEALTH Group. CONSORT-EHEALTH: improving and standardizing evaluation reports of Web-based and mobile health interventions. J Med Internet Res $2011 \mathrm{Dec}$ 31;13(4):e126. doi: 10.2196/jmir.1923.

72. Khoja S, Durrani H, Scott RE, Sajwani A, Piryani U. Conceptual Framework for Development of Comprehensive e-Health Evaluation Tool. Telemed J E Health 2013;19(1):48-53.

73. Call to Action on Global eHealth Evaluation Consensus Statement of the WHO Global eHealth Evaluation Meeting, Bellgio, September 2011; available from http://www.healthunbound.org/ content/call-action-global-ehealth-evaluation (accessed 31 January 2013).

74. Ham C, Hunter DJ, Robinson R. Evidence-based Policy; BMJ 1995;310(6972):71-2.

75. Walshe K, Rundall TG. Evidence-based Management: From Theory to Practice in Health Care. The Milbank Quarterly 2001;79:429-57.

76. Gray JAM. Evidence-based Healthcare: How to make Health Policy and Management Decisions. 2nd ed. Edinburgh: Churchill Livingstone; 2011. p. 444.

77. Rigby M, Ronchi E, Graham S. Evidence for building a smarter health and wellness future - Key messages and collected visions from a Joint OECD and NSF workshop. Int J Med Inform 2012 Oct 29. pii: S1386-5056(12)00195-5. doi: 10.1016/j. ijmedinf.2012.10.003. [Epub ahead of print].

Correspondence to:

Emeritus Professor Michael Rigby

Lavender Hill, 6 Carrighill Lower

Calverstown, Kilcullen

Co. Kildare, Ireland

Tel: +35345485858

E-mail:m.j.rigby@keele.ac.uk 